\title{
Smart Parking System: A Smarter Way of Parking Based on Ultrasonic sensors, NodeMCU, MQTT.fx
}

\author{
D.O.I - 10.51201/12490 \\ https://doi.org/10.51201/Jusst12490
}

\author{
Suresh Limkar ${ }^{1}$, Deep Patadiya ${ }^{1}$, Yash Gathani ${ }^{2}$, Abhishek Gaikwad ${ }^{1}$ \\ ${ }^{1}$ Department of Computer Engineering, AISSMS IOIT, Pune-01, India \\ ${ }^{2}$ Department of Computer Engineering, AISSMS COE, Pune-01, India
}

\begin{abstract}
This paper focuses on developing a smart parking system based on IOT. In many metropolitan cities, people are always worried whether they will find a parking slot or not. Even, finding an empty parking slot is very time con- suming and wastes a lot of fuel. After parking, to pay the required charges, a paper receipt is given and so there is a large amount of paper wasted every day. Thus, we have proposed a system consisting of ultrasonic sensors, NodeMCU deployed on-site and an android application available for the user. The android application will help the user to check the availability of a parking slot and reserve it. This will save time, reduce fuel consumption and thereby pollution.
\end{abstract}

Keywords: MQTT.fx, Ultrasonic sensors, NodeMCU, Parking slot, Quick Re- sponse (QR) code, Android app, MySQL database.

\section{Introduction}

In the past couple of years, the Internet of Things (IoT) has taken a boom in the indus- try. Today IoT is everywhere. Internet of Things (IoT) is a network of physical devic- es like sensors, software, and other devices that communicate and exchange data via the Internet. Suresh V. Limkar et al. [1] IoT is used in various sectors like transport, utilities, smart cities, healthcare, agriculture, etc. IoT integration can help automate several parts of smart cities. One of them is parking. In today's world, finding a park- ing space is becoming increasingly exhaustive each day. Pampa [2] says that it takes about 15 minutes to find a parking slot which increases fuel wastage, air pollution, traffic congestion, and futile efforts of the driver. Manickam et al. [3], D. Vakula et al.

[4] suggest that this parking problem increases more in holidays and festive seasons, and chances of accidents get higher. Sometimes, the user needs a parking administra- tor or parking staff to guide them to the parking slot. Some places also use receipts or a sticker which increases paper wastage and again requires manual intervention. Ratnadira et al. [5] says that human intervention shows how our current parking sys- tem is ineffective because parking slot information needs to be gathered manually. There should be as little manual work as possible. Sanam et al. [7] says sometimes the user is not aware the parking is legal or not. There are also times when there is no parking space available. So, the user tends to park in the wrong place or the no- 
parking zone. This may lead to towing or penalty charges of no parking. Hanisha et al. [10] says that people also tend to spend a lot of time to find a safe parking spot. Sanam et al. [7] says that this improper parking and crowding of vehicles causes in- convenience for people walking on the road or footpath. The finding process is very time consuming and tiring. Gayatri et al. [13] says that sometimes frustrated drivers take up the parking slot of people with special needs. This parking problem also in- creases fuel wastage and air pollution concerns. Rishi et al. [11] says that due to this parking problem there is a wastage of time and the pollution due to this also increases carbon emission. Sanam et al. [14] says that the traditional parking system has no slot restoration option. Dharmini et al. [15] says that the increase in vehicles needs the traditional system to become more efficient.

Thus, for the above problems, we need a smart parking system. The smart parking can be a solution to minimize user's time, reduce fuel wastage as well as save the overall money for the fuel burnt in search of the parking space.

By implementing efficient smart parking system it will lead to following benefits:

- It can be used to organize the parking space efficiently.

- It will save the time of the user which he might waste in searching for a parking slot.

- It can help in reducing the fuel wastage in search of the parking slot thereby reducing pollution.

- Because of less fuel wastage, it will also save the environment from harmful pollu- tants.

- It will help the user by navigating the user to the parking spot through maps (GPS).

- It will reduce paper wastage (receipts) by maintaining the details through apps.

- Less human intervention will be there as the system is

automated. In short it will use optimum time, parking space,

fuel, etc.

\section{$2 \quad$ Literature Review}

Pampa [2] proposed a system which consists of real-time parking detection, estimat- ing vehicle duration in slot, automated price calculation over the city. It uses several IoT components like ultrasonic sensors, LEDs, alarms, microcontrollers, cameras, solar panels, battery, and Wi-Fi modules for parking slots. The IoT components are connected to a Wi-Fi access point which is connected to the local server. These local servers are connected to the central server. It helps the user to find the parking slot throughout the city, and also calculates the price automatically based on the dura- tion. The user is not navigated to the parking and the maintenance cost of the system is high. The proposed method helps the user to find the parking slot from his android smartphone throughout the city but does not navigate the user to the slot. The compo- nents used in the system increases the maintenance cost of the system.

Manickam et al. [3] has proposed a cloud-based smart parking system using 
IoT to help the user find the nearest slot. It uses Arduino with NodeMCU and ultrasonic sensors for checking whether the car is present or not in the slot and also updates parking details accordingly. The user gets the details of the nearest parking slot avail- able from the app. It is cloud-based so it helps the user to access the data through the internet. The user is not navigated to the parking slot and there is no reservation of the slot. The proposed method is short and simple and can be implemented for specific areas only.

D.Vakula et al. [4] has proposed a smart parking system that uses IoT modules and an online platform for booking the parking slots. It uses Arduino with NodeMCU and ultrasonic sensors for checking the slot availability and has an online portal for book- ing parking slots. It lets the user book a parking slot through the interface provided which is easy and simple to understand. The proposed method is simple and cost ef- fective but there is a manual generation of the receipt which can be considered as wastage of the paper.

Ratnadira et al. [5] has proposed a system that uses the IoT module, an application for parking management and user and also uses crowd sensing. It uses ultrasonic sen- sors, Arduino and Ethernet for connection purposes. It uses sensors based on granularity of the parking slot and can adjust the cost according to it. It tries to adjust cost based on the parking granularity for saving cost on sensors, but this affects the overall system.

C. Ajchariyavanich et al. [6] has proposed a system that uses IoT with cloud inte- gration for the smart parking system. It uses ultrasonic sensors for car detection in the slot, NodeMCU for the internet connectivity, parking flap + servo-meter for slot entry and QR code reader for payment purposes. It is safe as no car can be parked unless the flap is open. The system has many parts and so the maintenance cost is high. It uses a flap for slot entry, so if the slot gets damaged then someone may park inappro- priately or in the wrong slot.

Sanam et al. [7] has proposed a system that uses an android application to detect the nearby slot and assign them digitally and also direct the user to the parking slot. It uses Pete (photon-enhanced thermionic emission) sensors that use solar energy. These sensors will help to detect if parking slots are vacant or not using the WSN method. The system has a multilingual android app. It has sensors that use solar energy, so if the weather changes, there can be several issues with the sensors and if they get dam- aged, the replacement cost will be high.

Pranav et al. [8] has proposed a system that uses a web app or mobile app to check slot availability with cloud connectivity. It has IR/ultrasonic sensors that are used based on the parking slot location (indoor / outdoor). It uses temperature sensors to turn on sprinklers in case any fire hazard occurs, light sensors for automatically turn- ing the lights on/off based on darkness, Arduino mega is used for controlling these sensors and cloud connectivity is used to show the slot details. The system is simple but not cost-effective and also increases maintenance cost as it uses a lot of sensors.

Meenaloshini.M et al. [9] has proposed a system that can book a slot using an app that will save the users time in search of the parking slot and reduce fuel consumption and unwanted travelling in search of parking slot. It focuses on a vertical parking system and uses infrared sensors for car detection in the parking slot. These sensors are connected to the app through IoT which makes it faster. It also has an LCD screen for users without the app. The infrared sensors increase the maintenance cost of the system as they are expensive.

Hanisha et al. [10] has proposed a system which has different modules like parking availability calculation, proximity estimation and payment service. It uses Raspberry- pi-3 connected to the cloud for receiving parking details. The 
user has to enter car details and will be allocated the nearest slot using Dijkstrae"s algorithm and will be navigated to the slot. While entering the parking, the license plate will be captured using image processing and IR sensors will detect the car. The time will be calculated and the user has to pay the total amount. It also has Led indication based on slot res- ervation. The proposed method calculates proximity based on user location within a specific radius which gives a proper idea of the nearby slots. It uses image processing on license plates by using cameras and IR sensors for slot detection. This makes the system less cost-effective and increases the maintenance cost.

Rishi et al. [11] has proposed a system that gives an overall system utility by using an android app for getting slot details and reserving a slot through it. It uses ultrasonic sensors for car detection in slots that are connected to Arduino Uno which is connect- ed to the Raspberry-pi-3. As the user books the slot they get a QR code in the app which they have to scan at the parking entrance. The QR code is used for uniquely identifying user for the reserved slot. The proposed system is simple and easy to un- derstand but does not have an administration system for controlling the overall sys- tem.

Norah et al. [12] has proposed a system that provides multiple facilities, including searching of parking slots, slot reservation, payment, receiving notifications, showing statistics, and monitoring parking states. It applies concepts of the Internet of Things (IoT) by using supportable hardware and sensors to detect parking availability and recognition to control access processes. It does not have timer limit which can cause unnecessary blocking of slot in case a car does not arrive and also it has multiple hardware requirements such as ANPR camera which increases the cost of the model.

Gayatri et al. [13] has proposed an IoT-based smart parking system which is more advantageous than the traditional parking system. Raspberry-pi-3 is used to store the information provided by local units located at each car park. It helps in reserving the parking spot in advance which helps in reducing time for searching the parking spots, traffic congestion, pollution, etc. Multiple hardware requirements such as RFID read- ers, Control unit and IR sensor will increase the cost. The user will have to search the address manually as no GPS feature is provided. Also, the role of application interface for traffic police is not mentioned.

Sanam et al. [14] has proposed a system that shows the parking problem can be tackled with a well-devised plan. An android application is used to store user details and GPS technology to track the nearest parking area making parking systems smart- er, convenient, reliable and efficient in booking of the parking slot. It mentions the need of a supervisor to monitor the area, hence not serving much advantage over a traditional parking system.

Dharmini et al. [15] has proposed a system that makes the traditional concept of parking system smarter by leveraging the power of IoT and embedding it with the latest innovation of electronic sensors and computers. The details are stored in cloud 
storage, IP cameras are used for detecting number plate and sensors are connected to Raspberry-pi which is then connected to the database. It provides more accommoda- tion of vehicles, user-friendly parking, less power consumption and facilitates more efficient use of space. It needs centralized database for user identification which can cause the system to slow down and adds restrictions to the system. No GPS function- ality is provided and IP cameras increase the overall cost.

Nawsheen et al. [16] has proposed a smart android-based parking-control applica- tion which will help you to find out a parking area nearby your location. It uses an android application for GUI purposes and MySQL database for storing data. PHP is used for connection purposes. Parking time can be calculated effectively. Involvement of shopkeeper is needed for the marking of unoccupied parking space and to stop the timer. Thus the system provides very few benefits as no shopkeeper can monitor his parking spot frequently.

\section{Proposed System}

In this paper we have proposed a smart parking system which helps in reducing the unnecessary efforts required to find a parking slot by giving the user an app through which he/she can book the slot.

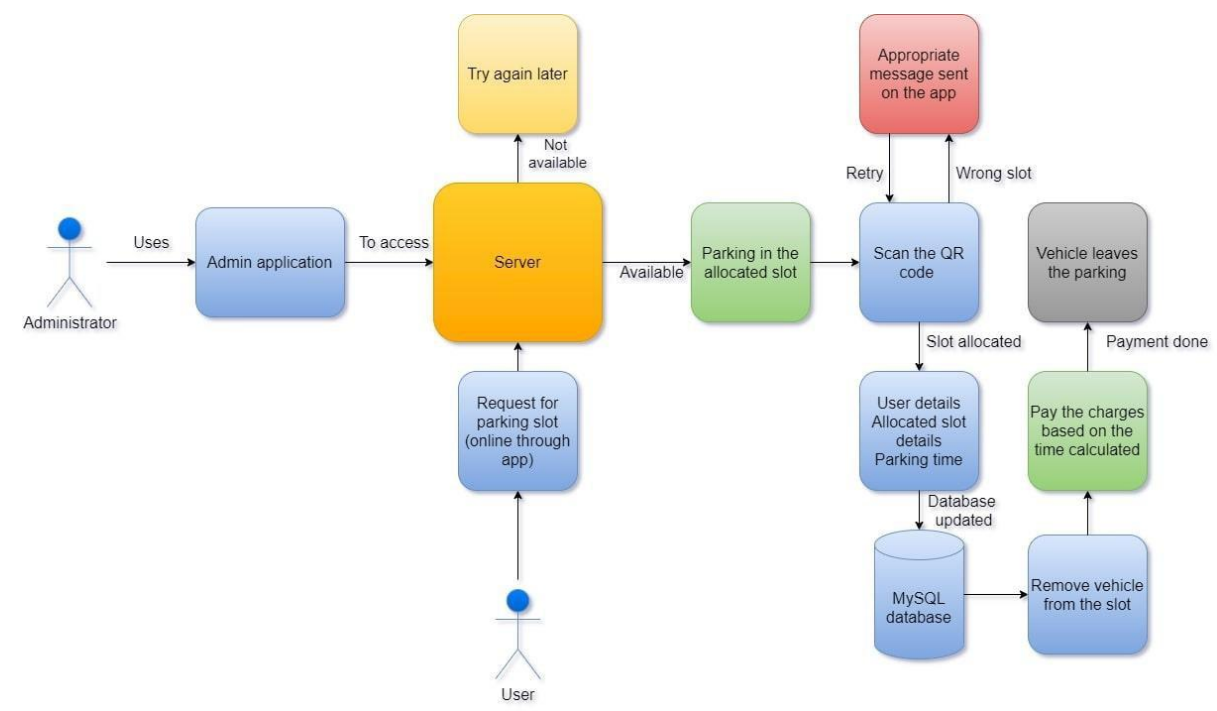

Fig. 1. System Architecture

The user will first open and log into the android application or if he is a new user, he will have to register first. The user can then search for a parking slot. If the parking slot is available, the user will be assigned a parking slot and it will be reserved for him. The user will also be navigated from the current location to the parking slot, using the application. After reserving the parking slot, if the user does not park the 
vehicle within 30 minutes, then that slot reserved for him will be cancelled. The de- tailed procedure is shown in Fig. 1.

After parking the vehicle, user has to scan the "QR code sticker" in the parking slot itself via application camera. The slot allocated to the user will be accessed from the database and it will be matched with the slot number of the sticker. If it does not match then the user will be notified:

"Allocated slot number: $x^{\text {ee }}$

"Vehicle parked in slot number: $y^{\text {ee, }}$ which is depicted in Fig. 2.

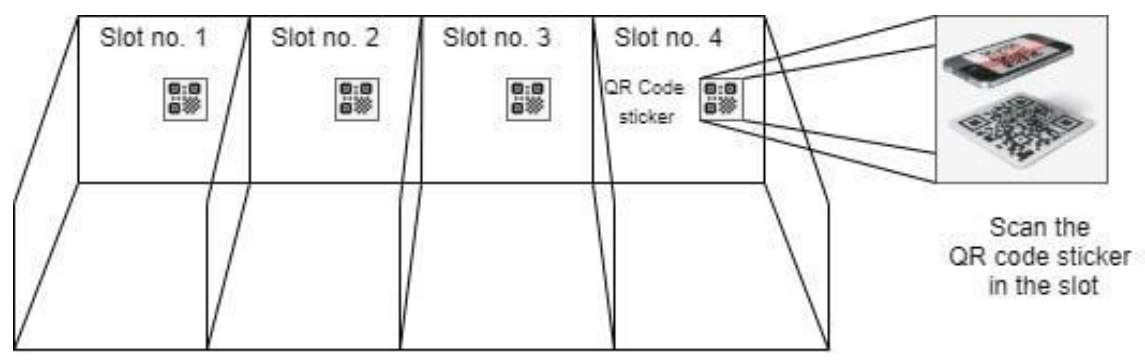

Fig. 2. QR code scanning process

After parking in the slot properly, the charges will be calculated according to the time the car was there in the parking slot. The calculation price for slot can be decided by the owner of the parking area. The user can link his bank account or UPI and pay the appropriate charges. The application will also have a feature to track the history, where the user will be shown his history of previous parking and the charges paid by the user each time.

There is also an admin app for the admin of the parking. So the admin can check the parking details and can also change the pricing for slots and the reserved timing for slots, i.e. the time in which the user has to park the car in the slot, according to his or her convenience.

Algorithm:

1. The user opens the android application.

2. The user requests for parking slot.

3. If slot $==$ available then

slot_available $=$ true, i.e. User can reserve the slot.

Else

slot_available $=$ false, i.e. User has to try again later.

4. The user will be navigated to the parking slot through Google maps.

5. After reserving the slot, the user has to park the car in the slot within a given timeframe.

6. If the user does not park within the timeframe

then Go to step 2, i.e. the user has to book a

new slot. Else

Go to step 7, i.e. the user proceed to the next step. 
7. After parking the vehicle, the user has to scan the QR sticker available in the park- ing slot.

8. If qr_sticker_data $==$ user_alloted_slot_number then

Go to step 9, i.e. Parking time and calculation of the charges

will start. Else

An appropriate message will be shown on the app to park in the correct slot and has to repeat step 7.

9. After removing the car form the slot, the user will have to pay the charges through the app which are calculated based on the time the car was parked in the slot.

10. The user can leave the parking.

\section{Software Architecture}

The software architecture of the system contains: an Android app, a Server, MySQL, MQTT.fx.

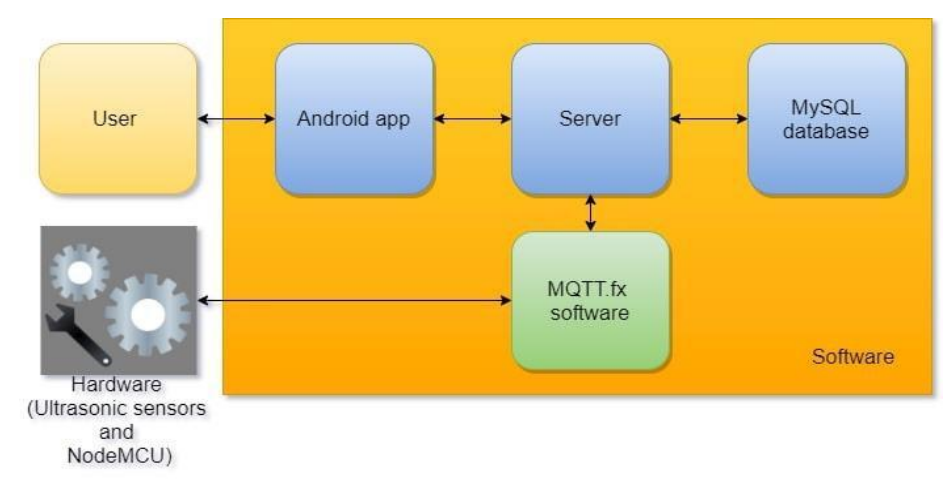

Fig. 3. Software

architecture

The user uses the android app on the mobile to communicate with Java server which is deployed on the heruko cloud, i.e. to check of the availability of the parking slot and reserve if it is available. The Java server then communicates with the MySQL database to check the availability of slot and sends appropriate response back to the user and also updates the database for the slot details. The database also helps to keep track of all slots in the parking area and also the details of every user, as shown in Fig. 3.

\section{Hardware Architecture}

The hardware architecture of the system contains: Ultrasonic sensors, NodeMCU. 


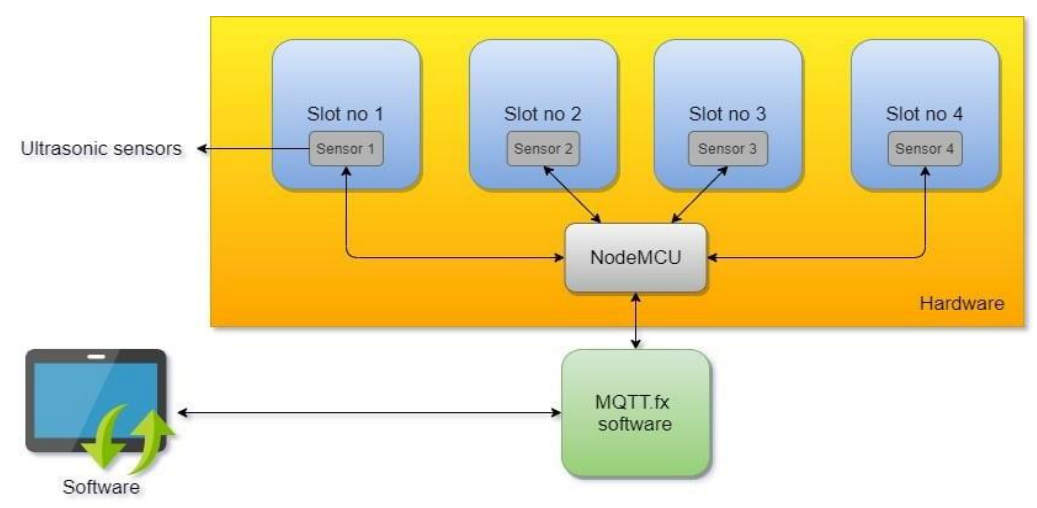

Fig. 4. Hardware architecture

The ultrasonic sensors are used to check is there a vehicle present in the parking slot or not. These ultrasonic sensors are connected to the NodeMCU which acts as the controller for the sensors and checks the status of the sensors.

The NodeMCU is also connected to the MQTT.fx software which acts an interface between NodeMCU and Java server. The Java server requests details of the parking slot availability through MQTT.fx. One NodeMCU can handle up to 3-4 ultrasonic sensors, as shown in Fig. 4.

\section{Details of the modules}

The details about modules in the system are as follows:

\section{- Android app :}

It is used by the user to communicate with the Java server to check slot availability, reserve a slot, do payments and keep a track of the history as well.

\section{- Server :}

It is a Java server. It handles the operations of checking the slot availability, slot reservation, updating database and updating the user accordingly. The server is hosted on the cloud. It communicates with the Android application, MQTT.fx software and also the MySQL database.

\section{- MySQL database :}

It used to maintain the details about all the slots i.e. the slots reserved slots empty and slots full. It also maintains the in and out time of the user which helps in calculat- ing the charges. It stores the detail of every user that has registered on the application.

\section{- MQTT.fx software [17] :}

MQTT (MQ Telemetry Transport or Message Queuing Telemetry Transport) is a lightweight, publish-subscribe network protocol that transports messages between devices. MQTT.fx is MQTT client based on JavaFX. For the proposed system, it acts as an interface between Java server and NodeMCU.

\section{- Ultrasonic sensors [18] :}

It is an electronic device which measures the distance of a target object by produc- ing ultrasonic sound waves, and converting the reflected sound into an electrical sig- 
nal. For the proposed system, the ultrasonic sensors are used for each parking slot to check the presence of the vehicle.

\section{- NodeMCU [19] :}

NodeMCU is a low-cost open source firmware and development kit platform that helps to build IoT products. For the proposed system, NodeMCU acts as a controller for the ultrasonic sensors and communicates with the MQTT.fx software.

\section{$4 \quad$ Result and Discussion}

The result of the proposed system adds multiple benefits over the traditional parking system. The time-consuming problem of searching and navigating to the parking slot is solved. As an effort to reduce environmental problems fuel wastage is also reduced and online payment eliminates the need for parking receipts. Therefore we can say that the system provides a very efficient, environmentally friendly solution to tackle the rising parking problem in the cities.

\section{Conclusion}

The problems which arise in the day to day life of people during parking a vehicle can be solved using the smart parking system. The above proposed system provides a cost effective solution for many general as well as environmental problems. It saves the time of people for searching a parking slot to park their vehicle. When searching for a parking slot, there is a lot of fuel wasted and thus this has a harmful impact on envi- ronment. However, as the user reserves a slot prior and is also navigated to the slot, fuel will not be wasted in search for a parking slot. There is less human intervention as everything is done with the help of an android application. The parking charges can also be paid through the app and so there is no need for receipts, thus saving paper. Therefore, smart parking system is necessary to eradicate the above mentioned prob- lems.

\section{References}

1. Suresh V. Limkar, Rakesh Kumar Jha, A novel method for parallel indexing of real time geospatial big data generated by IoT devices, Future Generation Computer Sys- tems, Volume 97,2019,Pages 433452,ISSN 0167-739X, https://doi.org/10.1016/j.future.2018.09.061.

2. P. Sadhukhan, "An IoT-based E-parking system for smart cities," 2017 International Con-ference on Advances in Computing, Communications and Informatics (ICACCI), Udupi, 2017, pp. 1062-1066, doi: 10.1109/ICACCI.2017.8125982. 
3. M. Ramasamy, S. G. Solanki, E. Natarajan and T. M. Keat, "IoT Based Smart Parking System for Large Parking Lot," 2018 IEEE 4th International Symposium in Robotics and Manufacturing Automation (ROMA), Perambalur, Tamil Nadu, India, 2018, pp. 1-4, doi: 10.1109/ROMA46407.2018.8986731.

4. D. Vakula and Y. K. Kolli, "Low cost smart parking system for smart cities, " 2017 Interna- tional Conference on Intelligent Sustainable Systems (ICISS), Palladam, 2017, pp. 280- 284, doi: 10.1109/ISS1.2017.8389415.

5. R. Widyasari, M. Z. Catur Candra and S. Akbar, "IoT-based Smart Parking System Devel- opment," 2019 International Conference on Data and Software Engineering (ICoDSE), Pontianak, Indonesia, 2019, pp. 1-6, doi: 10.1109/ICoDSE48700.2019.9092707.

6. C. Ajchariyavanich et al., "Park King: An IoT-based Smart Parking System," 2019 IEEE International Smart Cities Conference (ISC2), Casablanca, Morocco, 2019, pp. 729-734, doi: 10.1109/ISC246665.2019.9071721.

7. S. Kazi, S. Khan, U. Ansari and D. Mane, "Smart Parking based System for smarter cit- ies," 2018 International Conference on Smart City and Emerging Technology (ICSCET), Mumbai, 2018, pp. 1-5, doi: 10.1109/ICSCET.2018.8537281.

8. P. Chippalkatti, G. Kadam and V. Ichake, "I-SPARK: IoT Based Smart Parking System," 2018 International Conference On Advances in Communication and Computing Technol- ogy (ICACCT), Sangamner, 2018, pp. 473-477, doi: 10.1109/ICACCT.2018.8529541.

9. M. Meenaloshini, J. Ilakkiya, P. Sharmila, J. C. Sheffi Malar and S. Nithyasri, "Smart Car Parking System in Smart Cities using IR," 2019 3rd International Conference on Compu- ting and Communications Technologies (ICCCT), Chennai, India, 2019, pp. 178-182, doi: 10.1109/ICCCT2.2019.8824953.

10. H. Jamtani, M. Shah, K. Vanvari and S. Sahu, "IOT based Parking Automation System," 2018 International Conference on Smart City and Emerging Technology (ICSCET), Mum- bai, 2018, pp. 1-5, doi: 10.1109/ICSCET.2018.8537252.

11. R. Gupta, S. Pradhan, A. Haridas and D. C. Karia, "Cloud Based Smart Parking System," 2018 Second International Conference on Inventive Communication and Computational Technologies (ICICCT), Coimbatore, 2018, pp. 341-345, doi: 10.1109/ICICCT.2018.8473084.

12. N. Farooqi, S. Alshehri, S. Nollily, L. Najmi, G. Alqurashi and A. Alrashedi, "UParking: Developing a Smart Parking Management System Using the Internet of Things," 2019 Sixth HCT Information Technology Trends (ITT), Ras Al Khaimah, United Arab Emir-ates, 2019, pp. 214218, doi: 10.1109/ITT48889.2019.9075113.

13. G. N. Hainalkar and M. S. Vanjale, "Smart parking system with pre \& post reservation, billing and traffic app," 2017 International Conference on Intelligent Computing and Con- trol Systems (ICICCS), Madurai, 2017, pp. 500-505, doi: 10.1109/ICCONS.2017.8250772.

14. S. Kazi, S. Nuzhat, A. Nashrah and Q. Rameeza, "Smart Parking System to Reduce Traffic Congestion," 2018 International Conference on Smart City and Emerging Technology (ICSCET), Mumbai, 2018, pp. 1-4, doi: 10.1109/ICSCET.2018.8537367. 
15. D. Kanteti, D. V. S. Srikar and T. K. Ramesh, "Smart parking system for commercial stretch in cities," 2017 International Conference on Communication and Signal Processing (ICCSP), Chennai, 2017, pp. 1285-1289, doi: 10.1109/ICCSP.2017.8286588.

16. N. Promy and S. Islam, "A Smart Android Based Parking System to Reduce the Traffic Congestion of Dhaka City," 2019 21 st International Conference on Advanced Communica- tion Technology (ICACT), PyeongChang Kwangwoon_Do, Korea (South), 2019, pp. 124- 128, doi: 10.23919/ICACT.2019.8701935.

17. [Online available on: 09/11/2020] https://steemit.com/utopianio/@tanvirrahman/learn-about-mqtt-fx

18. [Online available on: 09/11/2020] https://www.fierceelectronics.com/sensors/what- ultrasonic-sensor

19. [Online available on: 09/11/2020] https://cityos-air.readme.io/docs/esp8266nodemcu 